\title{
Human Umbilical Cord Perivascular Cells Exhibited Enhanced Migration Capacity towards Hepatocellular Carcinoma in Comparison with Bone Marrow Mesenchymal Stromal Cells: A Role for Autocrine Motility Factor Receptor
}

\author{
Juan Bayo, ${ }^{1}$ Esteban Fiore, ${ }^{1}$ Jorge B. Aquino, ${ }^{1,2}$ Mariana Malvicini, ${ }^{1}$ Manglio Rizzo, ${ }^{1}$ \\ Estanislao Peixoto, ${ }^{1}$ Laura Alaniz, ${ }^{1,2}$ Flavia Piccioni, ${ }^{1}$ Marcela Bolontrade, ${ }^{2,3}$ \\ Osvaldo Podhajcer, ${ }^{2,3}$ Mariana G. Garcia, ${ }^{1,2}$ and Guillermo Mazzolini ${ }^{1,2,4}$ \\ ${ }^{1}$ Laboratorio de Terapia Génica, Facultad de Ciencias Biomédicas, Universidad Austral, B1629ODT Derqui-Pilar, \\ Buenos Aires, Argentina \\ ${ }^{2}$ CONICET (Consejo Nacional de Investigaciones Científicas y Técnicas), C1033AAJ Buenos Aires, Argentina \\ ${ }^{3}$ Laboratorio de Terapia Molecular y Celular, Fundación Instituto Leloir, C1405BWE Buenos Aires, Argentina \\ ${ }^{4}$ Unidad de Hígado, Hospital Universitario Austral, Universidad Austral, B1629ODT Derqui-Pilar, Argentina \\ Correspondence should be addressed to Mariana G. Garcia; mggarcia@conicet.gov.ar and \\ Guillermo Mazzolini; gmazzoli@cas.austral.edu.ar
}

Received 17 February 2014; Accepted 17 June 2014; Published 23 July 2014

Academic Editor: David Bernardo Ordiz

Copyright (C) 2014 Juan Bayo et al. This is an open access article distributed under the Creative Commons Attribution License, which permits unrestricted use, distribution, and reproduction in any medium, provided the original work is properly cited.

\begin{abstract}
Hepatocellular carcinoma (HCC) is the third cause of cancer-related death worldwide. Unfortunately, the incidence and mortality associated with HCC are increasing. Therefore, new therapeutic strategies are urgently needed and the use of mesenchymal stromal cells (MSCs) as carrier of therapeutic genes is emerging as a promising option. Different sources of MSCs are being studied for cell therapy and bone marrow-derived cells are the most extensively explored; however, birth associated-tissues represent a very promising source. The aim of this work was to compare the in vitro and in vivo migration capacity between bone marrow MSCs (BMMSCs) and human umbilical cord perivascular cells (HUCPVCs) towards HCC. We observed that HUCPVCs presented higher in vitro and in vivo migration towards factors released by HCC. The expression of autocrine motility factor (AMF) receptor, genes related with the availability of the receptor on the cell surface (caveolin-1 and -2) and metalloproteinase 3, induced by the receptor activation and important for cell migration, was increased in HUCPVCs. The chemotactic response towards recombinant AMF was increased in HUCPVCs compared to BM-MSCs, and its inhibition in the conditioned medium from HCC induced higher decrease in HUCPVC migration than in BM-MSC. Our results indicate that HUCPVCs could be a useful cellular source to deliver therapeutic genes to HCC.
\end{abstract}

\section{Introduction}

Hepatocellular carcinoma (HCC) is one of the leading causes of cancer-related death worldwide. At early stages of the disease only a small fraction of patients are amenable to curative treatments that include surgical resection, liver transplantation, or radiofrequency ablation [1]. For patients with advanced HCC, the multikinase inhibitor sorafenib shows limited survival benefits in comparison with placebo
[2]. Thus, the development of new therapeutic approaches is critical, and cellular therapy emerges as a new potential strategy to deliver therapeutic genes to HCC.

Mesenchymal stromal cells (MSCs) constitute a heterogeneous population of cells that include adult multipotent cells [3]. MSCs are present in adult tissues and their involvement in repair mechanisms has been demonstrated as the result of their ability to migrate towards sites of injury, their capacity to differentiate into tissues of mesodermal origin (adipocytes, 
osteoblasts, and chondroblasts), and their immunoregulatory properties [4]. Moreover, the possibility of easily expanding these cells in vitro makes them useful tools for therapeutic use in regenerative medicine, immunomodulation purposes and also as cellular carriers for therapeutic genes [5].

MSCs can be isolated from different tissues. Among them, bone marrow- (BM-) derived stromal cells were the first described and are the most frequently used [6]. However, the requirement of invasive methods to obtain BM-MSCs is in support of using alternative sources such as adipose tissue, peripheral blood, and dental pulp [7]. In addition to these adult tissues, MSCs can be obtained from birth-associated tissues including placenta, amnion, and umbilical cord with the advantage of their availability avoiding the need of invasive procedures and eliminating other ethical concerns. Different types of MSCs have been obtained from the umbilical cord depending on if they were isolated from the whole umbilical cord (UC-MSCs), Wharton's Jelly (WJ-MSCs), the umbilical cord blood (CB-MSCs) [8], or perivascular areas (human umbilical cord perivascular cells, HUCPVCs) [9]. In particular, HUCPVCs may be advantageous candidates for cell therapy due to their lower donor variability, faster doubling time, and ready availability [10].

It has been demonstrated that MSCs from different sources display similar phenotypic and cytological characteristics [11-13]; however, differences in their functional role have also been previously described. For instance, BM and WJ-derived MSCs have different proliferative capacity [11] and secretome and proteomic profiles [14, 15].

The establishment and spread of a tumor is a process that involves a complex cross-talk between cancer cells and the tumor microenvironment. HCC cells were found to be modulated by the tumor milieu through the secretion of several factors, and the tumor cells modify their microenvironment in order to promote their own growth [16]. Particularly, MSCs express receptors for various cytokines and chemokines allowing them to migrate towards HCC tumors [17]. Thus, recruitment of MSCs to cancer microenvironment is likely mediated by the interaction of cytokines/chemokines and their specific receptors. As an example, it has been demonstrated that CXCR1 is involved in UC-MSC migration towards glioma [18] and that overexpression of CXCR1 [19] or CXCR4 in these cells [20] increases their homing into tumors. In addition, MSCs were engineered to express cytotoxic cytokines for treatment of lung tumors and non-Hodgkin's lymphomas [21, 22]. Thus, the possibility of increasing MSC migratory capacity and engraftment into tumors could enhance beneficial effects of therapeutic genes.

The aim of this work was to compare HUCPVCs and the "gold standard" BM-MSCs migratory capacity towards factors released from HCC and to analyze mechanisms therein involved.

\section{Materials and Methods}

2.1. Cell Lines. Human HCC cell line HuH7 was kindly provided by Professor Jesus Prieto (CIMA, University of Navarra, Pamplona, Spain) [23]. LX-2 cell line (human hepatic stellate cells generated by spontaneous immortalization in low serum conditions) was kindly provided by Dr. Scott Friedman (Division of Liver Diseases, Mount Sinai School of Medicine, New York, NY, USA) [24]. Human microvascular endothelial cells (HMEC-1) were from CDC (Centers for Disease Control, Atlanta, GA, USA) and WI-38 (human fibroblast cell line) from the American Type Culture Collection. Cell lines were cultured in complete DMEM $(2 \mu \mathrm{M}$ glutamine, $100 \mathrm{U} / \mathrm{mL}$ penicillin, $100 \mathrm{mg} / \mathrm{mL}$ streptomycin) and $10 \%$ heatinactivated fetal bovine serum (FBS). Primary culture of HCC cells (HC-PT-5) was previously generated in our laboratory [25]. The collection of the sample and the project was approved by the Institutional Evaluation Committee (CIE) from School of Biomedical Sciences, Austral University (Protocol no. 11-007), and written informed consent was obtained from the patient. HC-PT-5 was cultured up to 8 passages in 70\% DMEM/30\% F12 (Invitrogen/Life Technologies) culture medium supplemented with $2 \mu \mathrm{M}$ glutamine, $100 \mathrm{U} / \mathrm{mL}$ penicillin, $100 \mathrm{mg} / \mathrm{mL}$ streptomycin, and $10 \%$ FBS.

2.2. Isolation of BM-MSCs and HUCPVCs. BM-MSCs were obtained from healthy donors (Hospital Naval Pedro Mallo, Buenos Aires, Argentina) as described previously [25]. HUCPVCs were isolated from umbilical cord obtained from healthy donors at the Hospital Universitario Austral (Pilar, Buenos Aires, Argentina) using a protocol adapted from Sarugaser et al. [9]. In brief, umbilical cords were dissected and vessels with their surrounding Warthon's Jelly were pulled out. The perivascular mesenchymal tissue was removed from the vessels and mechanically disrupted. Minced fragments were plated in complete DMEM low glucose/20\% FBS (Internegocios S.A., Argentina). After 7-day incubation, nonadherent cells and minced fragments were removed and adherent HUCPVCs were cultured and used for different experiments at passages 4 to 6 .

MSCs were characterized according to the International Society for Cellular Therapy (ISCT) guidelines [26]. Phenotype characteristics of MSCs were determined by flow cytometry with anti-human PE conjugated antibodies against CD14, CD34, CD44, CD73, CD90, and CD105 (BD Biosciences) for $30 \mathrm{~min}$. Samples were analyzed using a FACSCalibur flow cytometer (Becton Dickinson), and data acquired were analyzed using Cyflogic software (CyFlo Ltd.).

2.3. Conditioned Medium. To obtain tumor conditioned medium (TCM), HuH7 cells $\left(2 \times 10^{6}\right)$ or HC-PT- 5 cells (5 $\times 10^{6}$ ) were inoculated subcutaneously (s.c.) into the right flank of nude mice. When tumors reached $200 \mathrm{~mm}^{3}$ in size approximately, tumors were dissected and minced into pieces smaller than $1 \mathrm{~mm}^{3}$ and transferred to a 24 -well tissue culture plate (6 fragments/well) with $500 \mu \mathrm{L}$ of complete DMEM without FBS. Cell conditioned medium (CCM) was obtained from cell lines cultured as described above. Then, cells that reached a $90 \%$ of confluence were washed with PBS and cultured with complete DMEM without FBS. In both cases, 18 hours later, conditioned media were harvested and stored at $-80^{\circ} \mathrm{C}$ until use. 
2.4. In Vitro Migration Assays. In vitro migration was performed using a 48-Transwell microchemotaxis Boyden Chamber unit (Neuroprobe, Inc.) as previously described [25]. MSCs $\left(1.2 \times 10^{3}\right.$ cells/well $)$ were placed in the upper chamber and DMEM, CCM, TCM, or rAMF were applied to the lower chamber of the transwell unit. For blocking experiments, TCM were preincubated for $60 \mathrm{~min}$ with antiAMF antibody or isotype control IgG. All systems were left at $37^{\circ} \mathrm{C}$ in a $5 \% \mathrm{CO}_{2}$ humidified atmosphere for 4 hours except for experiments involving rAMF that were maintained for 18 hours. Cells attached to the lower side of the membrane were fixed in $2 \%$ formaldehyde, stained with $4^{\prime}, 6$-diamidino2-phenylindole dihydrochloride (DAPI, Sigma-Aldrich), and counted using fluorescent-field microscopy at 100x. Captured images from three representative visual fields were analyzed using Cell Profiler software (http://www.cellprofiler.com/) and the mean number of cells/field \pm SEM was calculated.

2.5. Cell Adhesion Assays. For analyses of MSC adhesion to endothelial cells, $2 \times 10^{5}$ HMEC-1 were seeded in 96well microplates and cultured for 1 day prior to the assay. Coated wells were incubated for 5 minutes with $0.1 \mathrm{~mL}$ of $5 \times 10^{4}$ cells $/ \mathrm{mL}$ of Fast-DiO prelabelled MSCs. Cell suspension was discarded and attached cells were fixed with $2 \%$ paraformaldehyde. Cells were counted using fluorescentfield microscopy at 200x: pictures taken from ten representative visual fields were analyzed using Cell Profiler software (http://www.cellprofiler.com/) and the mean number of cells/field \pm SEM was calculated.

2.6. Reverse Transcription-Polymerase Chain Reaction (RT$P C R)$. Total RNA of MSCs was extracted using Trizol Reagent (Sigma-Aldrich Co., St. Louis, MO). Total RNA $(4 \mu \mathrm{g})$ was reverse-transcribed with $200 \mathrm{U}$ of SuperScript II Reverse Transcriptase (Invitrogen, Carlsbad, CA) using $500 \mathrm{ng}$ of Oligo (dT) primers. cDNAs were subjected to real-time polymerase chain reaction (qPCR) (Stratagene Mx3005p, Stratagene, La Jolla, CA, USA). For qRT-PCR, the mRNA levels of CXCR1, CXCR2, CCR2, IL-6 receptor (IL-6R), IL-6 signal transducer (IL-6ST), AMF receptor (AMFR), metalloproteinase 3 (MMP3), insulin-like growth factor-binding protein 3 (IGFBP3), caveolin-1 (CAV-1), and caveolin-2 (CAV-2) were quantified by SYBR Green (Invitrogen), using the following primers: CXCR1 forward $5^{\prime}$-TTTTCCGCCAGGCTTACCAT- $3^{\prime}$ and reverse $5^{\prime}$-AACACCATCCGCCATTTTGC-3'; CXCR2 forward 5'-TAAGTGGAGCCCCGTGGGG-3' and reverse $5^{\prime}$-TGGGCTCAGGGGCAGGATG-3'; CCR2 forward $5^{\prime}$-CGAGAGCGGTGAAGAAGTCA- $3^{\prime}$ and reverse $5^{\prime}$-AGCATGTTGCCCACAAAACC- ${ }^{\prime}$; IL-6R forward $5^{\prime}$-GCACTTGCTGGTGGATGTTC- $3^{\prime}$ and reverse $5^{\prime}$-AGCCTTTGTCGTCAGGGATG- $3^{\prime}$; IL-6ST forward $5^{\prime}$-CCCACCTCATGCACTGTTGA- $3^{\prime}$ and reverse $5^{\prime}$-TTATGTGGCGGATTCGGCTT3'; AMFR forward $5^{\prime}$-ACAAGATGTGGGCCTTGCAAGA- $3^{\prime}$ and reverse $5^{\prime}$-AAAACGCAGTGCTCCCAGGATA3'; MMP3 forward 5'-ACGCCAGCCAACTGTGATCCT$3^{\prime}$ and reverse $5^{\prime}$-ATATGCGGCATCCACGCCTGAA- $3^{\prime}$; IGFBP3 forward $5^{\prime}$-ACTGTGGCCATGACTGAG- $3^{\prime}$ and reverse $5^{\prime}$-AGAGTCTCCCTGAGCCTGA-3'; CAV-1 forward $5^{\prime}$-AATCCAAGCATCCCTTTGCCCA- $3^{\prime}$ and reverse $5^{\prime}$-ACCAGGCAGCTTTCTGTACGA-3'; CAV-2 forward $5^{\prime}$-GAGAGACAGGGGAGTTGTCAACTT- $3^{\prime}$ and reverse $5^{\prime}$-GCCCGGCCCAGAAATAATGAGAT-3'. PCR amplifications were carried out using a cycle of $95^{\circ} \mathrm{C}$ for 10 minutes and 45 cycles under the following parameters: $95^{\circ} \mathrm{C}$ for 30 seconds, $58^{\circ} \mathrm{C}$ for 60 seconds, $72^{\circ} \mathrm{C}$ for 30 seconds. At the end of PCR reaction, the temperature was increased from $60^{\circ} \mathrm{C}$ to $95^{\circ} \mathrm{C}$ at a rate of $2^{\circ} \mathrm{C} / \mathrm{min}$, and the fluorescence was measured every 15 seconds to construct the melting curve. Values were normalized to levels of glyceraldehyde-3-phosphate dehydrogenase (GAPDH; used as housekeeping) transcript (forward $5^{\prime}$-CATCTCTGCCCCCTCTGCTG- ${ }^{\prime}$ and reverse $5^{\prime}$ GCCTGCTTCACCACCTTCTTG-3'). Data were processed by the $\Delta \Delta \mathrm{Ct}$ method. The relative amount of the PCR product amplified from BM-MSCs was set as 1. A nontemplate control (NTC) was run in every assay, and all determinations were performed as triplicates in three separated experiments.

2.7. Mice and In Vivo Experiments. Six- to eight-weekold male nude mice $(\mathrm{Nu} / \mathrm{Nu})$ were purchased from CNEA (Comisión Nacional de Energía Atómica, Ezeiza, Buenos Aires, Argentina). Subcutaneous HuH7 tumors $\left(2 \times 10^{6}\right.$ cells $)$ were established and 10 days later BM-MSCs or HUCPVCs were intravenously (i.v.) injected. Tumor growth was assessed by calliper measurement, and tumor volume $\left(\mathrm{mm}^{3}\right)$ was calculated by the formula $\pi / 6 \times$ larger diameter $\times($ smaller diameter $)^{2}$. For in vivo migration studies, MSCs $\left(5 \times 10^{5}\right)$ were prelabeled with CM-DiI for histological analysis and DiR (Molecular Probes, Invitrogen) for fluorescence imaging (FI). FI was performed using the Xenogen In Vivo Imaging System (IVIS; Caliper Life Sciences, Hopkinton, MA, USA) 1 hour after MSC injection and every day until experimental end point. At day 3 mice were sacrificed and isolated tissues were exposed to FI. Images represent the radiant efficiency and were analyzed with IVIS Living Image (Caliper Life Sciences) software. Regions of interest (ROI) were automatically drawn around the isolated organs to assess the fluorescence signal emitted. For the total signal present in mice, results were expressed as total radiant efficiency in units of photons/second within the region of interest $[\mathrm{p} / \mathrm{s}] /\left[\mu \mathrm{W} / \mathrm{cm}^{2}\right]$. Signal present in tumor, liver, spleen, or lungs was expressed as percentage of total signal.

2.8. Detection of BM-MSCs by Fluorescence. To detect CMDiI+ cells within tumors, frozen sections were mounted in mounting media with DAPI (Vector Laboratories, Inc.) and observed under a fluorescence microscope at 200x.

2.9. Statistical Analyses. Unpaired 2-sided Student's $t$-test and one-way analysis of variance followed by posttests or KruskalWallis and Dunn's posttests (GraphPad Prism Software) were used for statistical analyses. $P$ values lower than 0.05 were considered as statistically significant. 

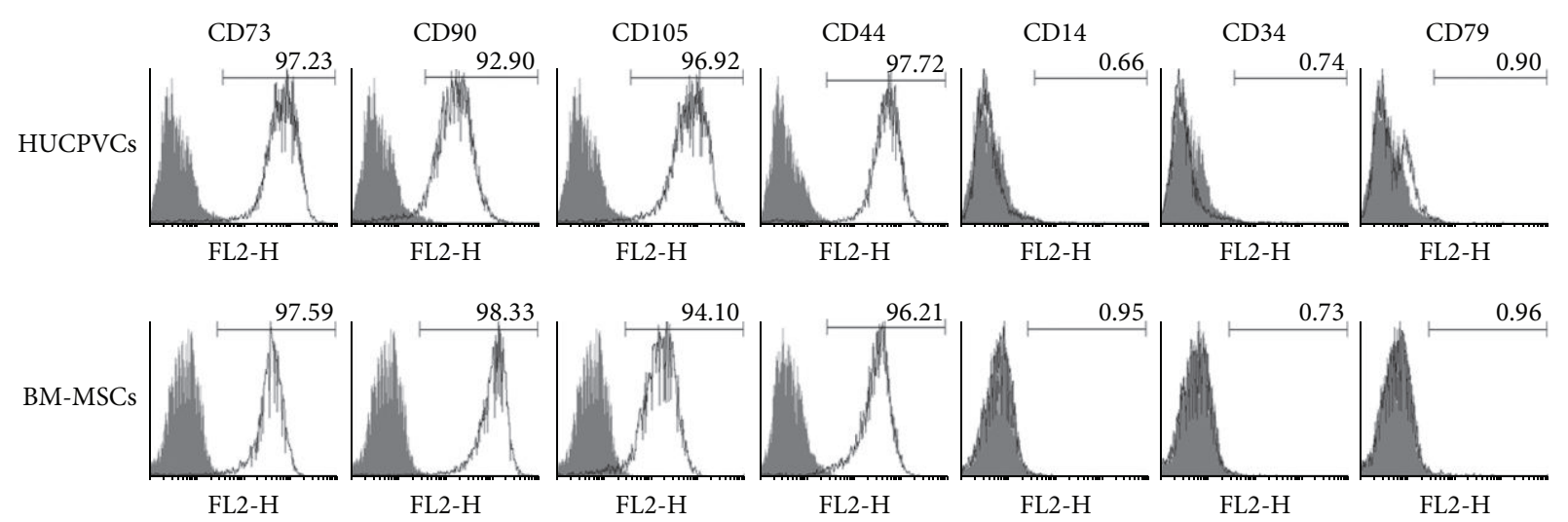

(a)

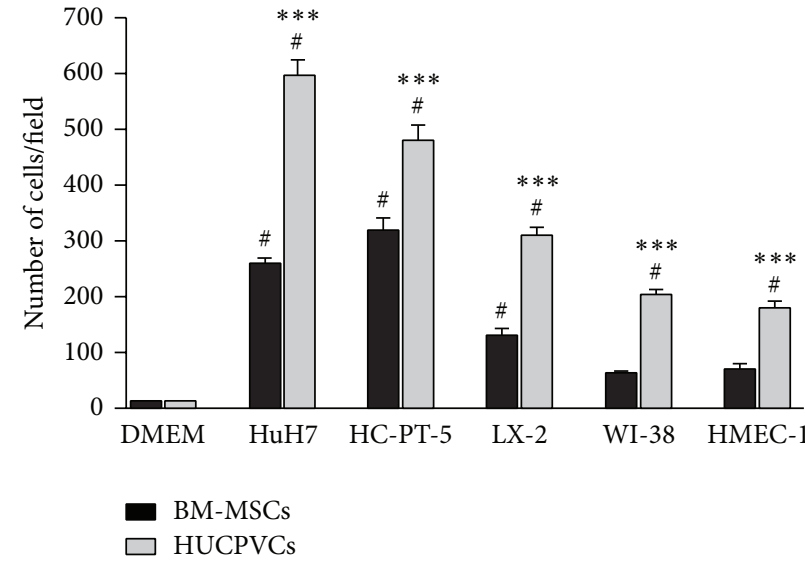

(b)

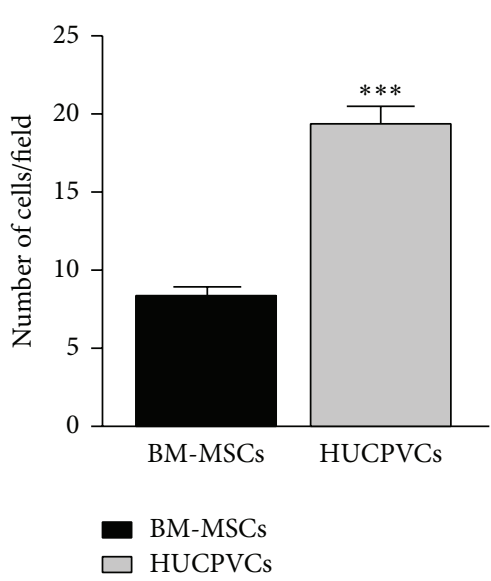

(c)

FIGURE 1: Characterization of BM-MSCs and HUCPVCs. (a) Flow cytometry analysis of cell surface markers of both types of MSCs. Grey area indicates background fluorescence with IgG isotype control. One representative experiment is shown. (b) In vitro migration of BM-MSCs (black bars) or HUCPVCs (grey bars) towards CCM from HCC (HuH7 and HC-PT-5), hepatic stellate cells (LX-2), fibroblasts (WI-38), or endothelial cells (HMEC-1). Bars represent the average of MSCs/field (10x) \pm SEM from three representative visual fields. Results are representative of 3 independent experiments. ${ }^{*} P<0.001$ versus DMEM; ${ }^{* * *} P<0.001$ versus BM-MSCs. (c) Adhesion towards endothelial cells of BM-MSCs (black bars) or HUCPVCs (grey bars). Results are representative of 3 independent experiments. ${ }^{* * *} P<0.001$ versus BM-MSCs.

\section{Results}

3.1. Characterization of BM-MSCs and HUCPVCs. In accordance with the criteria for defining MSCs of the International Society for Cellular Therapy (ISCT) [26], surface marker expression of BM-MSCs and HUCPVCs was evaluated by flow cytometry. Both types of MSCs showed similar phenotypic characteristics and were found to express CD73, CD90, CD105, and CD44 but not to express the hematopoietic markers CD14, CD34, or CD79 (Figure 1(a)). We next decided to evaluate the in vitro migration capacity of MSCs towards cell culture conditioned media (CCM) obtained from HCC cell lines (HuH7 and HC-PT-5), hepatic stellate cells (LX2), fibroblasts (WI-38), and endothelial cells (HMEC-1). Interestingly, a higher migratory capacity towards all the CCM was found for HUCPVCs when compared to BMMSCs (Figure 1(b)). Moreover, in contrast to our previous results observed with BM-MCSs [25], HUCPVCs showed capability to migrate to CCM derived from nontumoral components (fibroblast and endothelial cells). Besides their capacity to migrate toward factors secreted by HCC, the arrest of MSCs within the microvasculature is considered a critical step for an efficient homing and anchorage to tumors. Therefore, we next decided to evaluate adhesion ability of MSCs and observed that HUCPVCs showed an increased in vitro adhesion to HMEC-1 endothelial cells in comparison with BM-MSCs (Figure 1(c)).

3.2. In Vivo Migration of BM-MSCs and HUCPVCs towards HCC. To further characterize MSC behavior in vivo, noninvasive migration assay was performed. CM-DiI and DiR prelabelled BM-MSCs or HUCPVCs were i.v. injected in HCC tumor-bearing mice in order to evaluate MSC recruitment. Similar to our previous observation with BM-MSCs [25], at 3 days after cell transplantation a positive signal corresponding to HUCPVCs was found in liver, lungs, spleen, and s.c. tumors (Figure 2(a)). Despite the fact that total signal was 


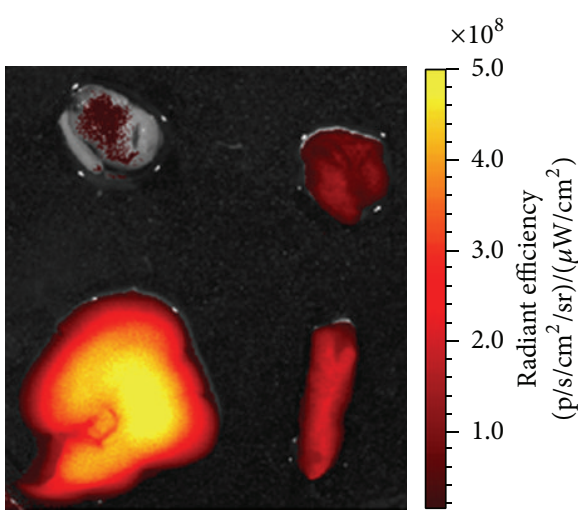

(a)

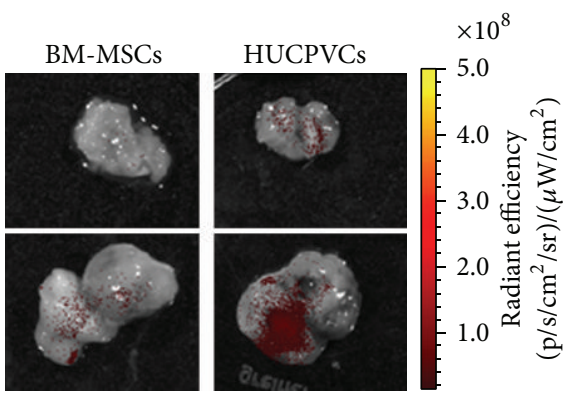

(c)

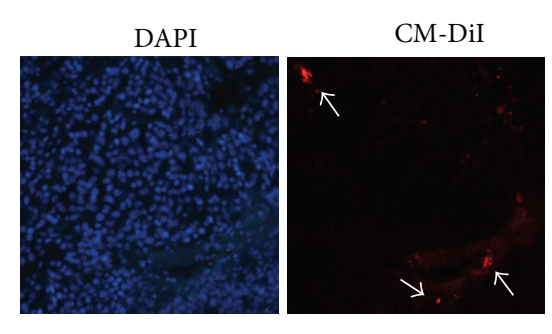

(e)

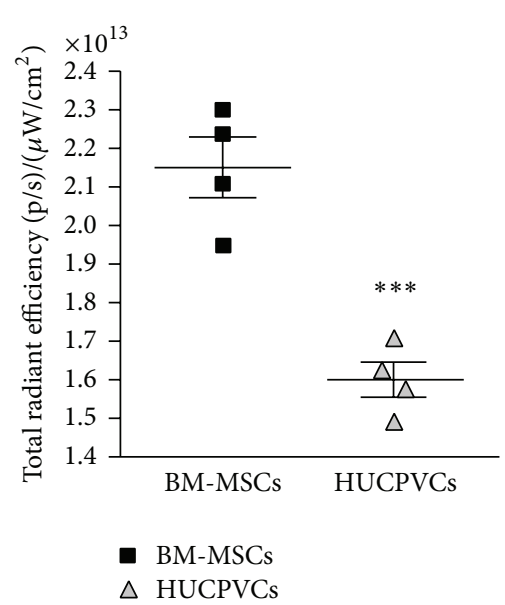

(b)

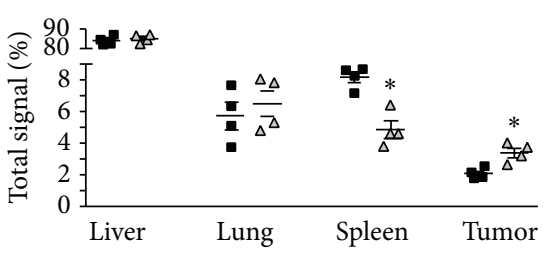

- BM-MSCs

$\triangle$ HUCPVCs

(d)

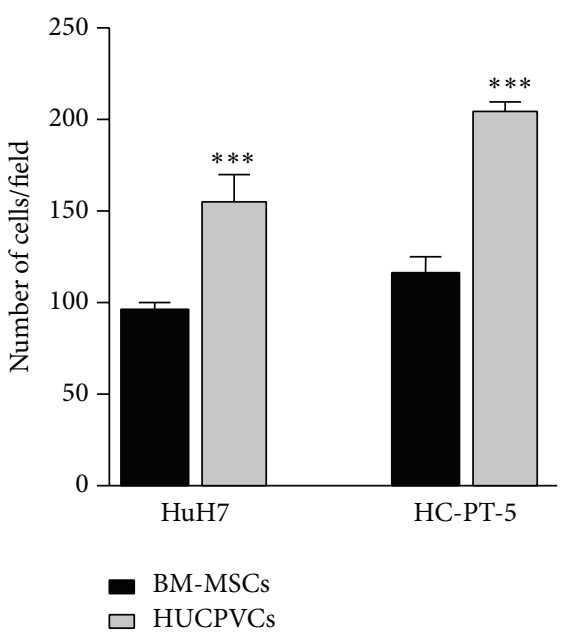

(f)

Figure 2: In vivo migration of BM-MSCs and HUCPVCs. CM-DiI and DiR prelabeled MSCs were i.v. injected in s.c. HuH7 tumor-bearing mice. At day 3 mice were sacrificed and organs were removed; lungs, livers, spleen (a) and tumors (c) were exposed to obtain FI. Images represent the radiant efficiency. Representative images are shown. (b) Total FI for injected BM-MSCs or HUCPVCs was calculated by measuring the region of interest (ROI) for all the tissues isolated and results were expressed as total radiant efficiency $[\mathrm{p} / \mathrm{s}] /\left[\mu \mathrm{W} / \mathrm{cm}^{2}\right]$. ${ }^{* * *} P<0.001$. (d) Signal present in the isolated liver, spleen, lungs and tumors was represented as percentage of total signal for BM-MSCs or HUCPVCs-injected mice. ${ }^{*} P<0.05$ versus BM-MSCs. (e) Microscopic analysis of transplanted CM-DiI-labeled MSCs (red signal indicated by arrows) and DAPI staining in frozen sections of tumors. $\times 200$ magnification. (f) In vitro migration of MSCs to TCM derived from HuH7 or HC-PT-5 s.c. tumors. Bars represent the average of MSCs/field (10x) \pm SEM from three representative visual fields. Results are representative of 3 independent experiments. ${ }^{* * *} P<0.001$ versus BM-MSCs. 


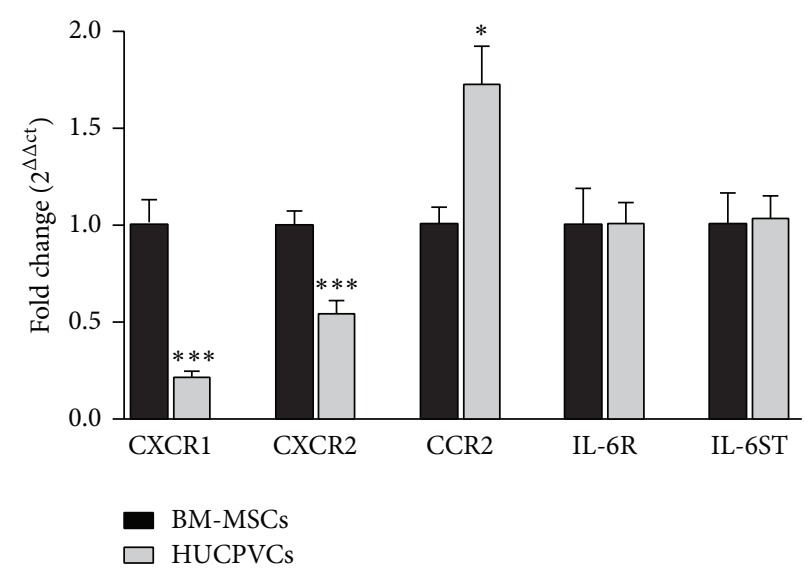

(a)

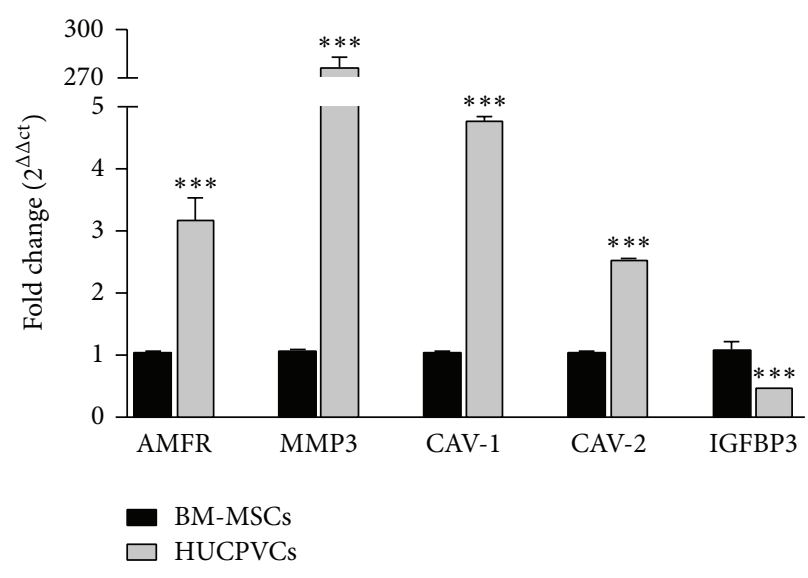

(b)

FIGURE 3: Differential expression of cytokines/chemokines receptors and AMF/AMFR pathway in MSCs. Expression of cytokines and chemokines receptors (a) and AMF/AMFR axis proteins (b) was evaluated in BM-MSCs (black bars) or HUCPVCs (grey bars) by qPCR. ${ }^{* * *} P<0.001$ versus BM-MSCs.

lower in mice injected with HUCPVCs compared to those injected with BM-MSCs (Figure 2(b)), the percentage of total signal corresponding to s.c. tumor locations was increased in mice administered with HUCPVCs in comparison with animals that received BM-MSCs (Figures 2(c) and 2(d)), indicating an enhanced engraftment of HUCPVCs into HCC tumors. In the other evaluated tissues, signal intensity was similar for BM-MSC or HUCPVCs in lung and liver and it was comparatively reduced in the spleen of HUCPVCsinjected mice (Figure 2(d)). Presence of MSCs in the s.c. tumors was also confirmed by fluorescence microscopy (Figure 2(e)). Finally, we evaluated whether MSCs might present differential migratory capacity towards $\mathrm{CM}$ obtained from s.c. tumors (TCM). Interestingly, a greater in vitro migratory capacity towards TCM from HCC was observed for HUCPVCs when compared to BM-MSCs (Figure 2(f)).

3.3. AMFR Was Highly Expressed in HUCPVCs. In order to evaluate mechanisms partially explaining the differential migratory capacity of HUCPVCs compared to BM-MSCs towards tumor released factors, we analyzed the expression of some chemokine's receptor likely involved in MSC recruitment towards HCC. Taking into account several reports demonstrating that interleukin- (IL-) 8, GRO, chemokine (C-C motif) ligand (CCL)-2, and IL-6 are among the most relevant factors in HCC [17], we decided to evaluate by qPCR the expression of CXCR1, CXCR2, CCR2, IL-6R, and IL6ST. Interestingly, constitutive CXCR1 and CXCR2 mRNA expression was found to be lower and CCR2 slightly higher in HUCPVCs when compared to BM-MSCs, while IL-6R and IL-6ST expression was similar in both MSCs sources (Figure 3(a)). Next, we decided to evaluate the axis of the autocrine motility factor (AMF), a cytokine with chemotactic effect on MSCs as recently observed by our group [27]. By qPCR, a significantly higher expression of the AMF receptor (AMFR) was found in HUCPVCs when compared to BM-MSCs. Similarly, genes known to be related to the availability of the receptor in the cell surface such as caveolin1 (CAV-1) and caveolin-2 (CAV-2) were also highly expressed in HUCPVCs as well as the metalloproteinase 3 (MMP3), necessary to the transmigration process. In contrast, expression levels of insulin-like growth factor-binding protein 3 (IGFBP3), a protein that negatively regulates AMF/AMFR pathway, were found to be reduced in HUCPVCs when compared to BM-MSCs (Figure 3(b)).

3.4. HUCPVCs Displayed Enhanced Migration towards AMF In Vitro. We have previously demonstrated that AMF, a cytokine produced by HCC cells, plays a critical role in MSC migration [27]. Due to the increased AMFR and AMFAMFR-related proteins expression in HUCPVCs, we decided to test the in vitro migration response to the recombinant AMF (rAMF) of both types of MSCs using a chemotaxis assay. A significantly higher migration to different doses of rAMF $(0.5$ and $0.75 \mu \mathrm{g} / \mathrm{mL})$ was observed for HUCPVCs when compared to BM-MSCs (Figure 4(a)). In spite of different types of MSCs showing similar reduction in migration levels (50\% of control) towards HuH7 TCM after the blockage with anti-AMF antibody (data not shown), preincubation of HCPT-5 TCM with anti-AMF antibody (AMF-ab) resulted in a further reduction in HUCPVCs migration capacity (54\% of control) when compared to BM-MSCs (67\% of control) (Figure 4(b)).

\section{Discussion}

The aim of this study was to assess the capability of different sources of MSCs to migrate towards HCC released factors in order to select those showing higher tumor recruitment capacity for future therapeutic applications. Our results indicated that although both types of MSCs share similar phenotypic characteristics as MSCs, HUCPVCs have a higher potential to migrate in vitro towards the conditioned medium derived not only from HCC cells, but also from 


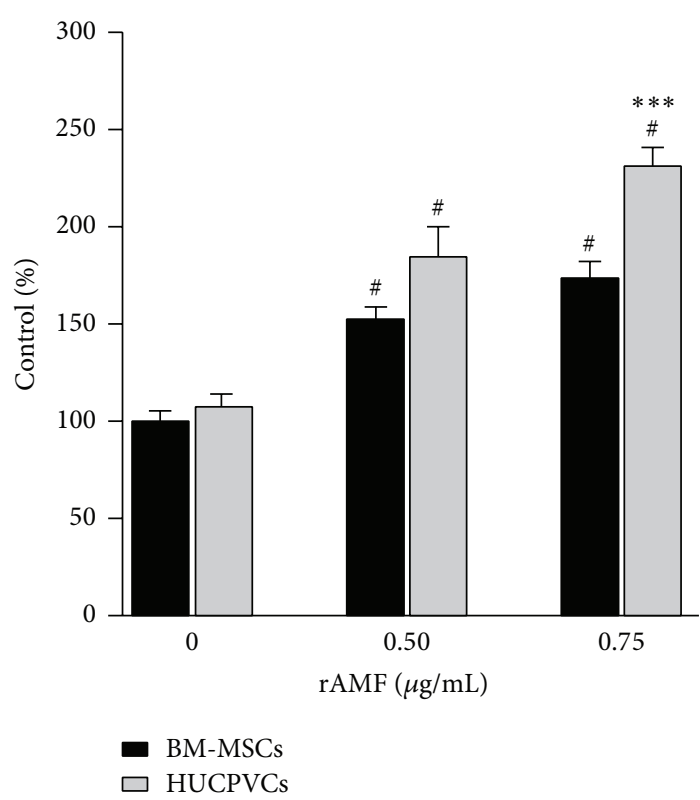

(a)

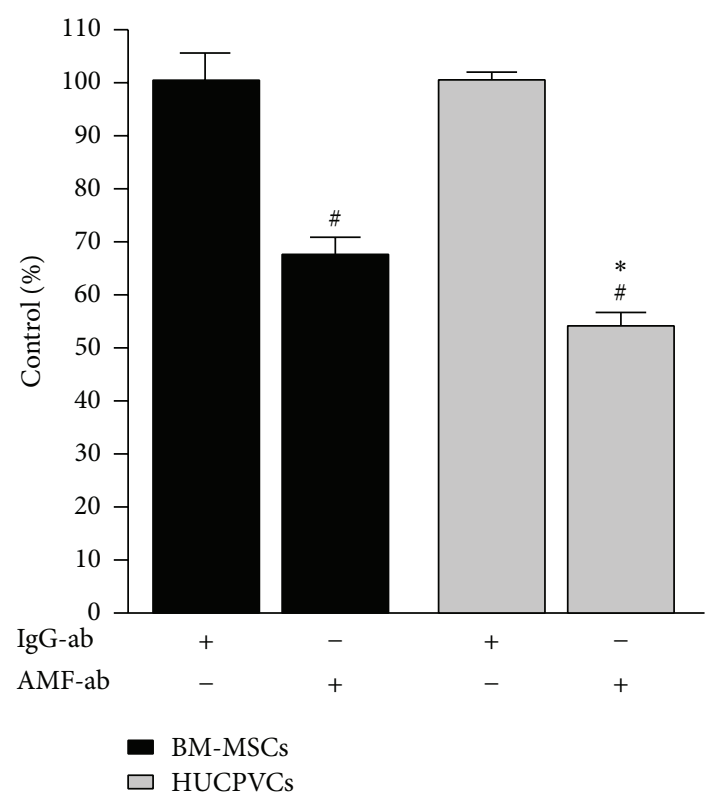

(b)

FIGURE 4: HUCPVCs showed enhanced migration towards AMF in comparison with BM-MSCs. (a) In vitro migration of BM-MSCs (black bars) or HUCPVCs (grey bars) towards rAMF. ${ }^{*} P<0.05$ versus DMEM $\left(0 \mu \mathrm{g} / \mathrm{mL}\right.$ rAMF); ${ }^{*} P<0.05$ versus BM-MSCs. (b) In vitro migration of BM-MSCs (black bars) or HUCPVCs (grey bars) towards HC-PT-5 TCM preincubated with anti-AMF antibody (AMF-ab) or control isotype (IgG-ab) was evaluated. ${ }^{\#} P<0.05$ versus IgG-ab; ${ }^{*} P<0.05$ versus BM-MSCs. Bars represent the average of MSCs/field (10x) \pm SEM from three representative visual fields. Results are representative of 3 independent experiments.

nontumoral components of the tumor microenvironment like activated hepatic stellate cells (LX-2), fibroblasts (WI38), or tumor microendothelial cells (HMEC-1). Moreover, HUCPVCs also showed an enhanced adhesion capacity to HMEC-1 cells compared to BM-MSCs. This enhanced adhesion to endothelial cells could allow a more efficient arrest in tumor microvasculature which is a required step for MSC recruitment into HCC. In line with this, we observed that HUCPVCs also exhibited greater migration capacity in vivo towards experimental HCC. In contrast to that observed in the in vitro migration experiments, HUCPVCs showed a similar migration capability towards nontumoral tissues like liver or lung and also showed a lower recruitment to the spleen. Although HUCPVCs migrated better than BM-MSCs toward activated hepatic stellate cells in vitro, no differences were observed in in vivo migration to normal liver, where hepatic stellate cells are quiescent. In addition, it should be noted that mice injected with HUCPVCs showed a lower total signal than those injected with BM-MSCs, probably due to differences in the DiR uptake for both kinds of cells or in the number of inoculated cells. These results are in agreement with previous works showing that MCSs derived from umbilical cord are able to migrate towards experimental tumors such as glioma [28], breast cancer [29], and lung adenocarcinoma [30]. Nonetheless, to the best of our knowledge, this is the first report describing the comparative migration capacity of human umbilical cord perivascular cells towards human HCC.
Tumor-homing is thought to be mediated by interactions of cytokines/chemokines with their specific receptors. A number of cytokines, growth factors, and chemokines are secreted by HCC cells and their microenvironment [17]. We have recently found that AMF is critical for MSC migration toward HCC in vitro and in vivo. Furthermore, other factors released by tumor stroma components like IL-8, GRO, IL-6, or CCL-2 could also be involved in MSC recruitment to HCC. Thus, differences observed between HUCPVCs and BMMSCs in their in vitro and in vivo migration capacity towards HCC seem at least partially related to differences in the expression of cytokine/chemokine receptors. Some studies have screened for similarities or differences among BMMSCs and UC-MSCs at the molecular level [31, 32]. In addition, a previous report demonstrated that migration of UCBMSC towards glioma cells was higher when compared to BM-MSCs likely due to increased levels of the IL-8 receptor, CXCR1, and CXCR2 in the former cell type [18]. In contrast to these observations, we found that HUCPVCs presented lower levels of CXCR1 and CXCR2 expression compared to BM-MSCs and these differences could be explained partially due to differences in tissue source and culture conditions and merit further investigation. The analysis of IL-6R and IL6-ST expression showed similar levels in both types of MSCs, while levels of MCP-1 receptor (CCR2) were slightly increased in HUCPVCs. Interestingly, we found that not only AMFR but also CAV-1 and CAV-2, which are genes that regulate the availability of AMFR on cell surface [33], were overexpressed in HUCPVCs compared to BM-MSCs. In line with this, a 
reduced expression level of IGFBP3, a protein which can bind to AMF blocking its binding to AMFR [34], was found in HUCPVCs when compared to BM-MSCs. Moreover, an increased expression of MMP3 was observed in HUCPVCs. This matrix metalloproteinase is involved in AMF-induced migration and it is necessary for cell invasion and proteolysis of the extracellular matrix [35]. Finally, and consistent with an increase in the expression of genes related to the AMF/AMFR pathway, not only HUCPVCs show an enhanced in vitro migration towards rAMF, but also the specific blockage of AMF in HC-PT-5 TCM was able to reduce HUCPVCs migration to a greater level when compared to BM-MSCs.

The potential use of MSCs in cancer treatment as carriers of therapeutic genes has raised some concerns about the safety of their use in the clinic. There are some studies that indicated that MSCs have protumorigenic capacity due to their immunosuppression properties, modulation of the epithelial-to-mesenchymal transition, and induction of angiogenesis [36]. However, others have demonstrated that MSCs decreased HCC growth [37, 38]. Similarly, we have previously demonstrated that BM-MSCs did not modify tumor growth in vivo [25]. Moreover, we observed that HUCPVCs did not induce tumor growth when injected i.v. in Huh7 s.c. tumor-bearing mice (data not shown). The use of HUCPVCs as cellular carriers could also have the advantage of its allogeneic application because of its low immunogenicity [9]. In this regard, HUCPVCs could be useful as carriers of therapeutic genes for cancer patients, where isolation of BM or adipose tissue-derived MSCs could be less responsive to chemotactic factors, and might display higher immunosuppression capacities than MSCs from healthy donors [39] or even present genetic abnormalities.

\section{Conclusions}

In summary, our results demonstrate a greater migration capacity to HCC of HUCPVCs when compared to BMMSCs not only in vitro but also in vivo, likely due to an increase in migratory response to AMF and to an enhanced adhesive capacity to tumor microvasculature. Considering their availability and that no invasive procedures are required to obtain HUCPVCs, these cells have advantages over BMMSCs as candidates for carriers of therapeutic genes for the treatment of HCC.

\section{Conflict of Interests}

The authors declare that there is no conflict of interests regarding the publication of this paper.

\section{Acknowledgments}

The authors gratefully thank Guillermo Gastón, Marcos Cabrera, and Vanina Ferreira for technical assistance and Dr. Mengshiao Meng (National Chung Hsing University (NCHU), Taichung, Taiwan) for providing recombinant AMF. This work was supported by Grants from Austral
University (for Juan Bayo I04-12; for Mariana G. Garcia T1312; and for Guillermo Mazzolini T13-11) and from Agencia Nacional de Promoción Científica y Tecnológica (ANPCyT) PICTO 2008/00115 (Mariana G. Garcia), PICT 2008/00123 (Jorge B. Aquino), PICTO 2008/00122 (Jorge B. Aquino), and PICT 2010/2818 (Mariana G. Garcia and Guillermo Mazzolini).

\section{References}

[1] H. B. El-Serag, J. A. Marrero, L. Rudolph, and K. R. Reddy, "Diagnosis and treatment of hepatocellular carcinoma," Gastroenterology, vol. 134, no. 6, pp. 1752-1763, 2008.

[2] J. M. Llovet, S. Ricci, V. Mazzaferro et al., "Sorafenib in advanced hepatocellular carcinoma," The New England Journal of Medicine, vol. 359, no. 4, pp. 378-390, 2008.

[3] A. J. Friedenstein, J. F. Gorskaja, and N. N. Kulagina, "Fibroblast precursors in normal and irradiated mouse hematopoietic organs," Experimental Hematology, vol. 4, no. 5, pp. 267-274, 1976.

[4] D. J. Prockop and J. Y. Oh, "Medical therapies with adult stem/progenitor cells (MSCs): a backward journey from dramatic results in vivo to the cellular and molecular explanations," Journal of Cellular Biochemistry, vol. 113, no. 5, pp. 1460-1469, 2012.

[5] J. B. Aquino, M. F. Bolontrade, M. G. García, O. L. Podhajcer, and G. Mazzolini, "Mesenchymal stem cells as therapeutic tools and gene carriers in liver fibrosis and hepatocellular carcinoma," Gene Therapy, vol. 17, no. 6, pp. 692-708, 2010.

[6] M. F. Pittenger, A. M. Mackay, S. C. Beck et al., "Multilineage potential of adult human mesenchymal stem cells," Science, vol. 284, no. 5411, pp. 143-147, 1999.

[7] H. Yoshimura, T. Muneta, A. Nimura, A. Yokoyama, H. Koga, and I. Sekiya, "Comparison of rat mesenchymal stem cells derived from bone marrow, synovium, periosteum, adipose tissue, and muscle," Cell and Tissue Research, vol. 327, no. 3, pp. 449-462, 2007.

[8] R. Hass, C. Kasper, S. Böhm, and R. Jacobs, "Different populations and sources of human mesenchymal stem cells (MSC): a comparison of adult and neonatal tissue-derived MSC," Cell Communication and Signaling, vol. 9, article 12, 2011.

[9] R. Sarugaser, D. Lickorish, D. Baksh, M. M. Hosseini, and J. E. Davies, "Human umbilical cord perivascular (HUCPV) cells: a source of mesenchymal progenitors," Stem Cells, vol. 23, no. 2, pp. 220-229, 2005.

[10] D. Baksh, R. Yao, and R. S. Tuan, "Comparison of proliferative and multilineage differentiation potential of human mesenchymal stem cells derived from umbilical cord and bone marrow," Stem Cells, vol. 25, no. 6, pp. 1384-1392, 2007.

[11] S. Kern, H. Eichler, J. Stoeve, H. Klüter, and K. Bieback, "Comparative analysis of mesenchymal stem cells from bone marrow, umbilical cord blood, or adipose tissue," Stem Cells, vol. 24, no. 5, pp. 1294-1301, 2006.

[12] O. K. Lee, T. K. Kuo, W. M. Chen, K. D. Lee, S. L. Hsieh, and T. H. Chen, "Isolation of multipotent mesenchymal stem cells from umbilical cord blood," Blood, vol. 103, no. 5, pp. 1669-1675, 2004.

[13] H. Wang, S. Hung, S. Peng et al., "Mesenchymal stem cells in the Wharton's jelly of the human umbilical cord," Stem Cells, vol. 22, no. 7, pp. 1330-1337, 2004. 
[14] J. Kim, J. M. Shin, Y. J. Jeon, H. M. Chung, and J. Chae, "Proteomic validation of multifunctional molecules in mesenchymal stem cells derived from human bone marrow, umbilical cord blood and peripheral blood," PLoS ONE, vol. 7, no. 5, Article ID e32350, 2012.

[15] J.-Y. Hsieh, H.-W. Wang, S.-J. Chang et al., "Mesenchymal stem cells from human umbilical cord express preferentially secreted factors related to neuroprotection, neurogenesis, and angiogenesis," PLoS ONE, vol. 8, no. 8, Article ID e72604, 2013.

[16] V. Hernandez-Gea, S. Toffanin, S. L. Friedman, and J. M. Llovet, "Role of the microenvironment in the pathogenesis and treatment of hepatocellular carcinoma," Gastroenterology, vol. 144, no. 3, pp. 512-527, 2013.

[17] J. Bayo, M. Marrodan, J. B. Aquino, M. Silva, M. G. Garcia, and G. Mazzolini, "The therapeutic potential of bone marrowderived mesenchymal stromal cells on hepatocellular carcinoma," Liver International, vol. 34, no. 3, pp. 330-334, 2014.

[18] D. Kim, J. H. Kim, J. Kwon Lee et al., "Overexpression of CXC chemokine receptors is required for the superior gliomatracking property of umbilical cord blood-derived mesenchymal stem cells," Stem Cells and Development, vol. 18, no. 3, pp. 511-519, 2009.

[19] S. M. Kim, D. Kim, C. H. Jeong et al., "CXC chemokine receptor 1 enhances the ability of human umbilical cord bloodderived mesenchymal stem cells to migrate toward gliomas," Biochemical and Biophysical Research Communications, vol. 407, no. 4, pp. 741-746, 2011.

[20] S. A. Park, C. H. Ryu, S. M. Kim et al., "CXCR4-transfected human umbilical cord blood-derived mesenchymal stem cells exhibit enhanced migratory capacity toward gliomas," International Journal of Oncology, vol. 38, no. 1, pp. 97-103, 2011.

[21] R. S. Rachakatla, F. Marini, M. L. Weiss, M. Tamura, and D. Troyer, "Development of human umbilical cord matrix stem cell-based gene therapy for experimental lung tumors," Cancer Gene Therapy, vol. 14, no. 10, pp. 828-835, 2007.

[22] C. Yan, S. Li, Z. Li et al., "Human umbilical cord mesenchymal stem cells as vehicles of CD20-specific TRAIL fusion protein delivery: a double-target therapy against non-Hodgkin's lymphoma," Molecular Pharmaceutics, vol. 10, no. 1, pp. 142-151, 2013.

[23] J. Hu, A. Dong, V. Fernandez-Ruiz et al., "Blockade of Wnt signaling inhibits angiogenesis and tumor growth in hepatocellular carcinoma," Cancer Research, vol. 69, no. 17, pp. 6951-6959, 2009.

[24] L. Xu, A. Y. Hui, E. Albanis et al., "Human hepatic stellate cell lines, LX-1 and LX-2: new tools for analysis of hepatic fibrosis," Gut, vol. 54, no. 1, pp. 142-151, 2005.

[25] M. G. Garcia, J. Bayo, M. F. Bolontrade et al., "Hepatocellular carcinoma cells and their fibrotic microenvironment modulate bone marrow-derived mesenchymal stromal cell migration in vitro and in vivo," Molecular Pharmaceutics, vol. 8, no. 5, pp. 1538-1548, 2011.

[26] M. Dominici, K. le Blanc, I. Mueller et al., "Minimal criteria for defining multipotent mesenchymal stromal cells: the International Society for Cellular Therapy position statement," Cytotherapy, vol. 8, no. 4, pp. 315-317, 2006.

[27] J. Bayo, E. Fiore, J. B. Aquino et al., "Increased migration of human mesenchymal stromal cells by Autocrine Motility Factor (AMF) resulted in enhanced recruitment towards hepatocellular carcinoma," PLoS ONE, vol. 9, no. 4, Article ID e95171, 2014.

[28] M. K. Seong, Y. L. Jung, I. P. Sang et al., "Gene therapy using TRAIL-secreting human umbilical cord blood-derived mesenchymal stem cells against intracranial glioma," Cancer Research, vol. 68, no. 23, pp. 9614-9623, 2008.

[29] R. Ayuzawa, C. Doi, R. S. Rachakatla et al., "Naïve human umbilical cord matrix derived stem cells significantly attenuate growth of human breast cancer cells in vitro and in vivo," Cancer Letters, vol. 280, no. 1, pp. 31-37, 2009.

[30] T. Matsuzuka, R. S. Rachakatla, C. Doi et al., "Human umbilical cord matrix-derived stem cells expressing interferon- $\beta$ gene significantly attenuate bronchioloalveolar carcinoma xenografts in SCID mice," Lung Cancer, vol. 70, no. 1, pp. 28-36, 2010.

[31] G. Li, X. Zhang, H. Wang et al., "Comparative proteomic analysis of mesenchymal stem cells derived from human bone marrow, umbilical cord, and placenta: implication in the migration," Proteomics, vol. 9, no. 1, pp. 20-30, 2009.

[32] H. C. Miranda, R. H. Herai, C. H. Thomé et al., "A quantitative proteomic and transcriptomic comparison of human mesenchymal stem cells from bone marrow and umbilical cord vein," Proteomics, vol. 12, no. 17, pp. 2607-2617, 2012.

[33] P. U. Le, G. Guay, Y. Altschuler, and I. R. Nabi, "Caveolin-1 is a negative regulator of caveolae-mediated endocytosis to the endoplasmic reticulum," The Journal of Biological Chemistry, vol. 277, no. 5, pp. 3371-3379, 2002.

[34] S. Mishra, A. Raz, and L. J. Murphy, "Insulin-like growth factor binding protein-3 interacts with autocrine motility factor/phosphoglucose isomerase (AMF/PGI) and inhibits the AMF/PGI function," Cancer Research, vol. 64, no. 7, pp. 25162522, 2004.

[35] F. L. Yu, M. H. Liao, J. W. Lee, and W. L. Shih, "Induction of hepatoma cells migration by phosphoglucose isomerase/ autocrine motility factor through the upregulation of matrix metalloproteinase-3," Biochemical and Biophysical Research Communications, vol. 314, no. 1, pp. 76-82, 2004.

[36] A. H. Klopp, A. Gupta, E. Spaeth, M. Andreeff, and F. Marini III, "Concise review: dissecting a discrepancy in the literature: do mesenchymal stem cells support or suppress tumor growth?" Stem Cells, vol. 29, no. 1, pp. 11-19, 2011.

[37] L. Qiao, Z. Xu, T. Zhao et al., "Suppression of tumorigenesis by human mesenchymal stem cells in a hepatoma model," Cell Research, vol. 18, no. 4, pp. 500-507, 2008.

[38] Y. R. Lu, Y. Yuan, X. J. Wang et al., "The growth inhibitory effect of mesenchymal stem cells on tumor cells in vitro and in vivo," Cancer Biology and Therapy, vol. 7, no. 2, pp. 245-251, 2008.

[39] Z.-G. Zhao, W. Xu, L. Sun, W.-M. Li, Q.-P. Li, and P. Zou, “The characteristics and immunoregulatory functions of regulatory dendritic cells induced by mesenchymal stem cells derived from bone marrow of patient with chronic myeloid leukaemia," European Journal of Cancer, vol. 48, no. 12, pp. 1884-1895, 2012. 


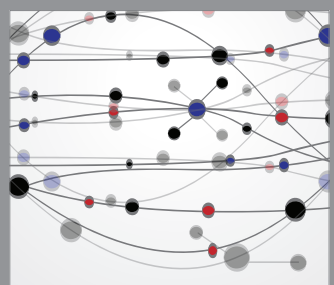

The Scientific World Journal
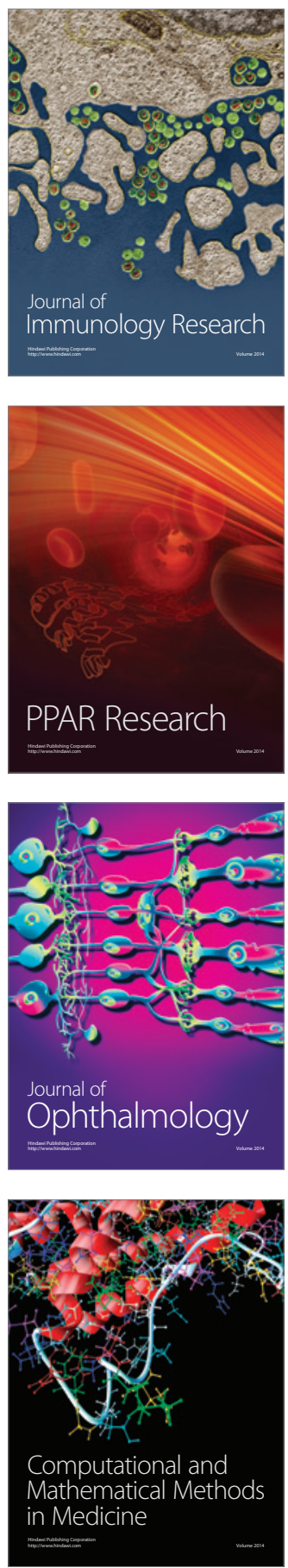

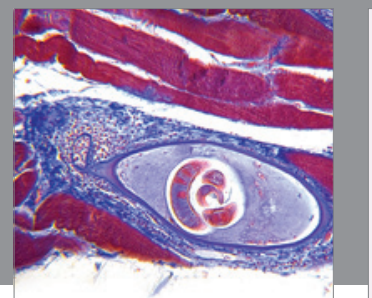

Gastroenterology

Research and Practice
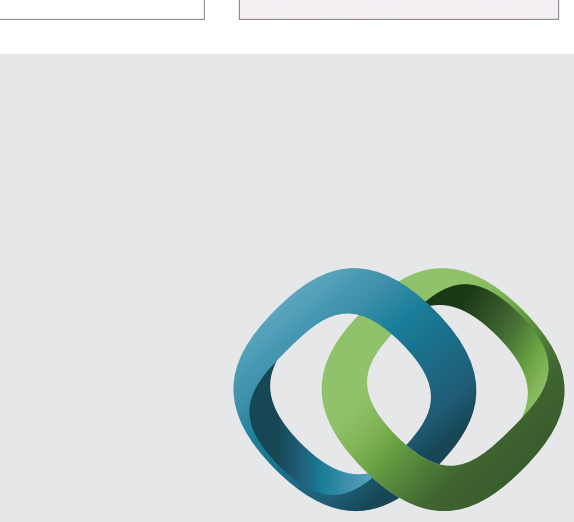

\section{Hindawi}

Submit your manuscripts at

http://www.hindawi.com
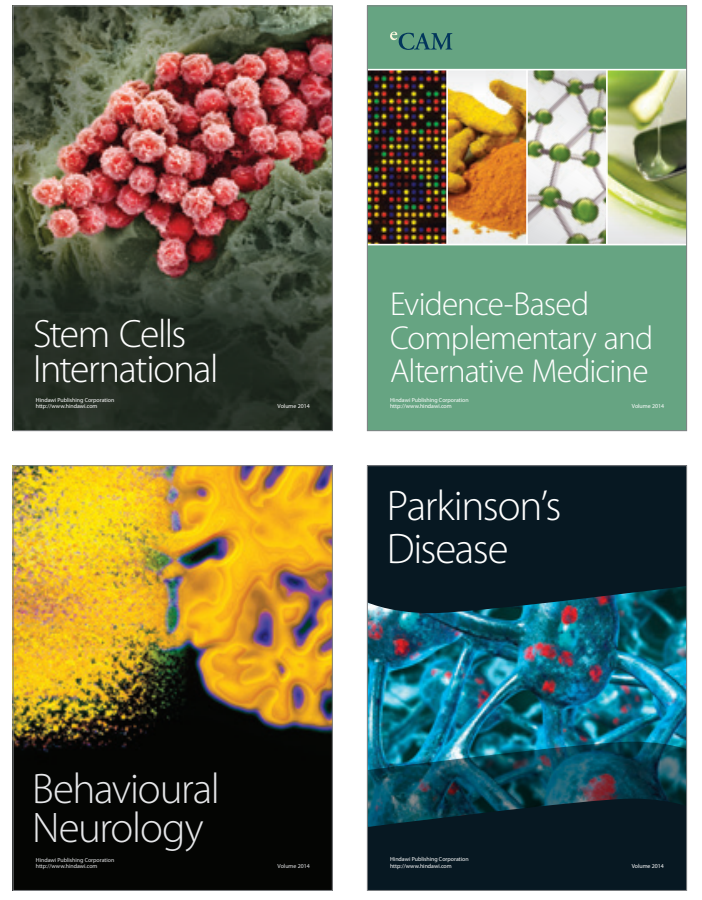
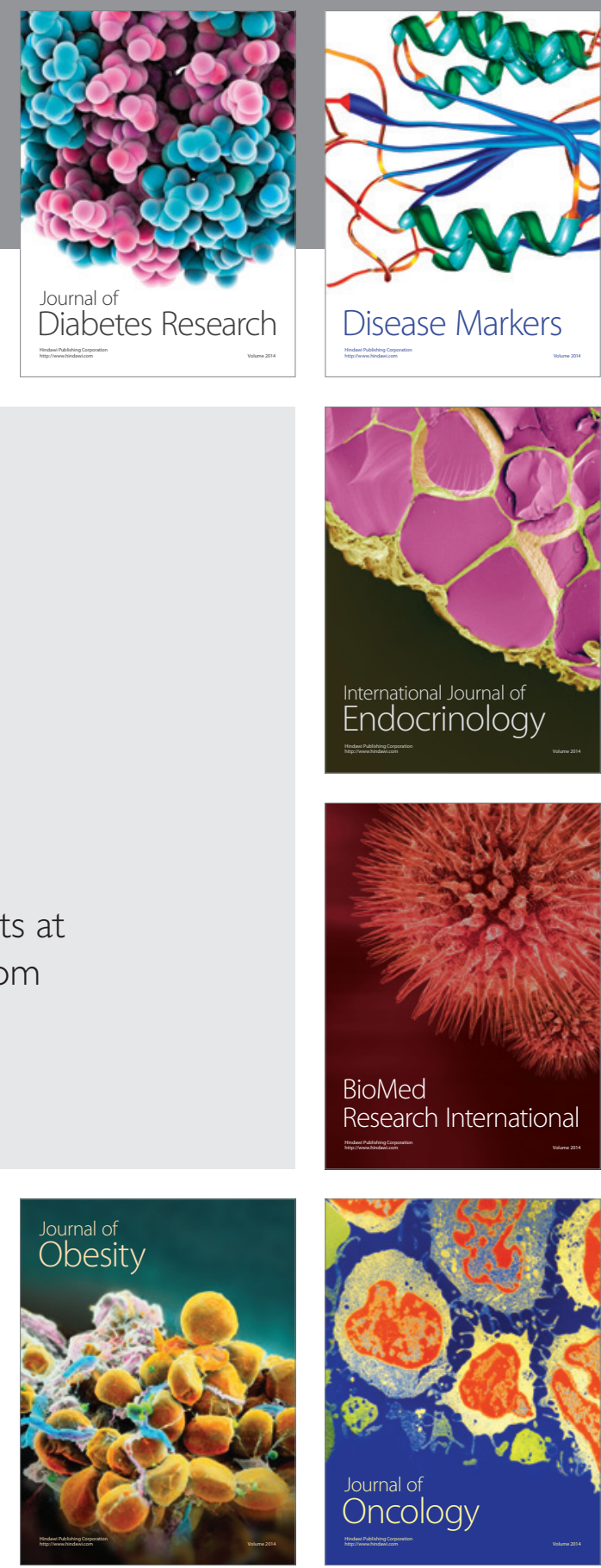

Disease Markers
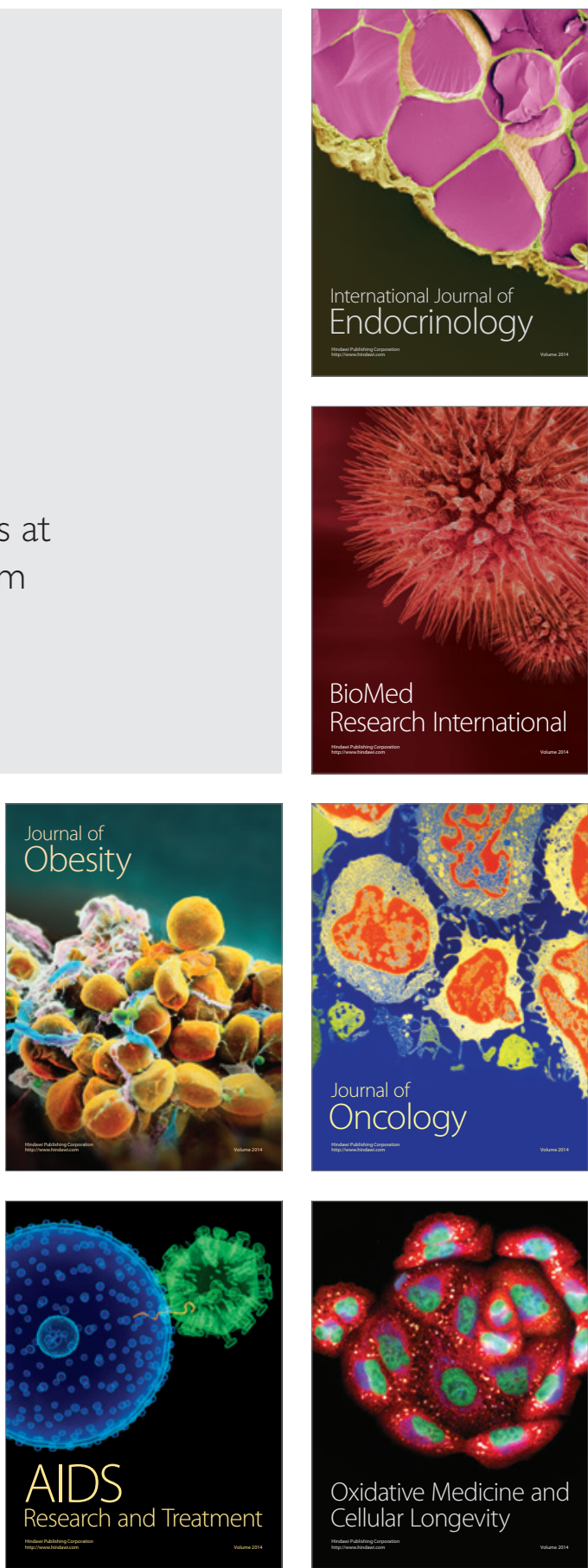